\title{
Vitamin C attenuates the toxic effect of nutmeg on primary visual occipital cortex in rats
}

\author{
N.A.A.F.A. Salman' ${ }^{1}$, F.E.-N.A.-H. El-Safty ${ }^{1}$, M.M. El-Habeby ${ }^{1}$, \\ W.B. El-Kholy', G.F.A. El-Akabawy',2 \\ ${ }^{1}$ Department of Anatomy and Embryology, Faculty of Medicine, Menoufia University, Menoufia, Egypt \\ 2Princess Nourah bint Abdulrahman University, Department of Basic Sciences, College of Medicine, Riyadh, Kingdom of Saudi Arabia
}

[Received: 9 May 2018; Accepted: 27 June 2018]

\begin{abstract}
Background: Nutmeg is neurotoxic in rats and possibly neurotoxic also in humans. The aim of this study is to investigate the effect of nutmeg on the primary visual occipital cortex of adult male rat and to evaluate the possible protective role of vitamin $C$.

Materials and methods: Fifty Sprague-Dawley adults male rats were randomly divided into three main groups; control, nutmeg-treated (500 and $1000 \mathrm{mg} / \mathrm{kg} /$ /day) and protected groups (nutmeg + vitamin C [500 mg/kg/day]). All rats were treated orally by gavage for 5 days per week for 6 weeks. At the end of the experiment, primary visual occipital cerebral cortex was subjected to histological, immunohistochemical and genetic analyses.

Results: Our results revealed toxic effects of nutmeg on the primary visual occipital cerebral cortex in adult male albino rat. This was indicated by histopathological alterations, including pyknotic nuclei surrounded with vacuolations by light microscopic studies and degenerations of organelles by electron microscopic studies. In addition, we detected an increase in immunoreactivity for GFAP and caspase-3 by immunohistochemical assessments. Apoptotic bands appeared in genetic studies. Co-administration of vitamin C ameliorated nutmeg-induced toxic alterations on the primary visual occipital cerebral cortex.

Conclusions: Nutmeg administration caused histopathological and genetic changes in the primary visual occipital cerebral cortex in adult male albino rats. These changes were improved by co-administration of vitamin C. (Folia Morphol 2019; 78, 1: 33-38)
\end{abstract}

Key words: antioxidant, apoptosis, cerebral cortex, neurotoxin, nutmeg, vitamin C

\section{INTRODUCTION}

Nervous system is considered one of the principal target systems in the body that could be attacked by a number of toxic substances, called neurotoxins. Neurotoxicity alters the normal activity of the nervous system and causes damage to it. Food additives, such as nutmeg can be neurotoxins [15]. Nutmeg originates from the fruit of the nutmeg tree, Myristica Fragrans, and is best known as the kitchen spice. It is used in the preparation of meat products, soups, sauces, baked foods, puddings, seasoning of vegetables and flavouring milk dishes and punches. It enters in the composition of numerous medicines, as that used to treat gastric disorders, rheumatism and also as 
a hypnotic and an aphrodisiac substance [3]. Because it is cheap and legal, this made it a popular narcotic substance among prisoners, seamen, soldiers and musicians. This spice may be grounded in Coca-Cola or milk [16]. At the same time, it has been long known for its neurotoxic effects and psychoactive properties of producing anxiety and fear [2]. Vitamin C can exert its neuroprotective role as a potent scavenger of oxygen free radicals [12]. The primary visual occipital cortex is the best-studied visual area in the brain. It is the simplest and the earliest cortical visual area. In addition, it is highly specialised for processing information about static and moving objects [9]. In spite of the above-mentioned data about nutmeg, its world-wide use and its reported neurotoxicity, not enough studies have been conducted to investigate its effect on primary visual occipital cortex. In addition, the possible protective role of vitamin $C$ against nutmeg-induced toxicity has not been evaluated.

\section{MATERIALS AND METHODS}

\section{Animals and experiment design}

This study was carried out on 50 adult male Sprague-Dawley albino rats, with an average weight of $200 \mathrm{~g}$. They were obtained from Helwan Animal House, Egypt. The animals were maintained in the animal house of the Faculty of Medicine, Menoufia University. The rats were subjected to a 12:12-h daylight/darkness and allowed unlimited access to chow and water. The procedure was approved by the ethics committee for animal experimentation of the Faculty of Medicine, Menoufia University in accordance with the international regulation on care and use of laboratory animals. The animals were randomly divided into three main groups: Control group A consisted of 18 rats; subdivided into three equal subgroups: sham subgroup A1 (received normal diet), vehicle subgroup A2 (received $1 \mathrm{~mL}$ distilled water, the same volume used to make nutmeg suspension), vitamin C-treated subgroup A3. Group B consisted of 18 rats; subdivided into two equal subgroups; subgroup B1 (low dose nutmeg-treated subgroup) and subgroup B2 (high dose nutmeg-treated subgroup). Group C consisted of 18 rats subdivided into two equal subgroups; subgroup C1 (low dose nutmeg + vitamin C-treated subgroup) and subgroup C2 (high dose nutmeg + vitamin C-treated subgroup).

\section{Chemicals}

Nutmeg powder was obtained from a supermarket in Egypt. It was administered at a dose of 500 and
$1000 \mathrm{mg} / \mathrm{kg}$ dissolved in $1 \mathrm{~mL}$ distilled water to make a suspension and was given by gavage 5 days/week for 6 weeks [1]. The toxic dose of nutmeg powder in humans is $5 \mathrm{~g}$. One tablespoonful of ground nutmeg spice is approximately equal to $7 \mathrm{~g}$ [14]. The equivalent dose of this toxic dose in rats is about $700 \mathrm{mg} /$ /kg [5]. In this study, two different doses were used, one lower and one higher than this value to examine if there was a dose-dependent toxicity of nutmeg. Vitamin C was purchased from a pharmacy in Egypt and was administrated at a dose of $500 \mathrm{mg} / \mathrm{kg} / \mathrm{day}$ by gavage 5 days/week for 6 weeks [11].

\section{Histological and immunohistochemical studies}

At the end of the experiment, each rat was anaesthetized using ketamine (90 $\mathrm{mg} / \mathrm{kg}$ ) and xylazine (15 mg/kg) (i.p.) and decapitated. Each brain was fixed in 10\% neutral buffered formalin and embedded in paraffin wax for histological examination. Semi-serial 5 coronal sections (1-in-20 series) were prepared from the occipital cortex and were dehydrated using ethanol and stained with haematoxylin and eosin (H\&E) for routine histological examination and also stained with toluidine blue for Nissl's granules. For immunohistological staining, paraffin sections ( $5 \mu \mathrm{m}$ thick) were deparaffinised in xylene and then subjected to immunohistochemical studies using caspase-3, 1:500, Labvision and glial fibrillary acidic protein [GFAP], Labvision. Other sections of the cerebral tissues were frozen and used for genetic studies by gel electrophoresis. Others were cut into about $1 \mathrm{~mm}$ specimens and fixed in $2.5 \%$ glutaraldehyde in $0.1 \mathrm{M}$ sodium phosphate buffer, $\mathrm{pH} 7.3$, for $3 \mathrm{~h}$ at $4 \mathrm{C}^{\circ}$, and routinely osmicated in $1 \%$ osmium tetroxide. After dehydration with graded ethanol series, the samples were embedded in Araldite. Semithin sections were stained with toluidine blue stain as a preliminary step.

\section{Quantitative assessments}

For histological and immunohistochemical quantitative assessment, 5 non-overlapping fields $(400 \times)$ per section were randomly captured by a Leica Microscope DML B2/11888111 equipped with a Leica camera DFC450. The number of immunopositive cells in the fields taken from at least three sections/animal was counted using ImageJ software (Maryland, USA) and averaged per field for each animal. The numbers calculated for at least 5 animals/experimental group were considered for comparison and statistical analyses. 


\section{Statistical analysis}

Results were expressed as mean \pm standard error of the mean (SEM). One way-ANOVA followed by a post hoc Bonferroni test was performed using GraphPad Prism version 5.00 for Windows, GraphPad Software, San Diego, California, USA. The level of significance of $p \leq 0.05$ was considered to be statistically significant.

\section{RESULTS}

\section{Histological results}

There was no significant difference between the control subgroups.

\section{Light microscopy}

Haematoxylin and eosin and toluidine blue studies. In H\&E-stained sections, the control group showed normal regular neurons; rounded granular and pyramidal nuclei with prominent nucleoli (Fig. 1A). Compared to control group, nutmeg-treated groups showed evidence of structural changes. These changes were more obvious in high dose nutmeg-treated cortices than that in low dose nutmeg-treated ones. The neurons, with their different sizes, exhibited damage. Some neurons were darkly-stained with pyknotic nuclei and surrounded by halos (Fig. 1B-D). Moreover, in subgroup B2, divided, shrunken and pale nuclei were detected (Fig. 1D). On the other hand, vitamin C-treated group showed a considerable maintenance of most of the normal architecture of the cerebral cortex. This was more observed in low dose nutmeg + vitamin C-treated subgroup (C1) than that in high dose nutmeg + vitamin C-treated subgroup (C2). Most of neurons were in normal shape and size, but a few neurons were still darkly-stained (Fig. 1E, F). In nutmeg-treated group, toluidine blue stained sections showed a decrease in the density of Nissl's granules in the cytoplasm of the neurons of its both subgroups compared to the control group. This reduction was more evident in high dose nutmeg-treated subgroup (B2) (Fig. 2C) than in low dose nutmeg-treated subgroup (B1) (Fig. 2B). The neurons of the protected group showed many normal neurons with darkly-blue stained cytoplasm containing numerous Nissl's granules.

Immunohistochemical studies. There was a significant increase in GFAP expression in nutmeg-treated subgroups compared with control group $A$ $(p<0.001)$. There was also a significant increase in GFAP expression in subgroup B2 compared with subgroup B1 $(p<0.001)$. Vitamin C co-treatment significantly inhibited nutmeg-induced increase in

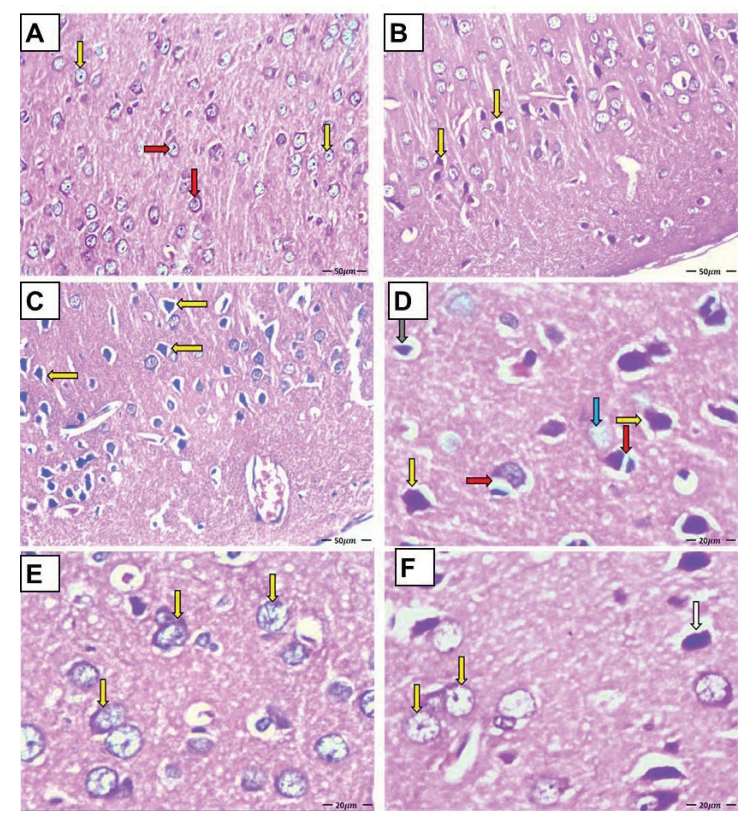

Figure 1. Representative images of haematoxylin and eosin (H\&E)-stained sections from rats of different groups. A. In group A, granular cells (yellow arrows) and pyramidal cells (red arrows) were seen. In subgroup B1, some pyknotic neurons (yellow arrows, B) were detected. In subgroup B2, most of nuclei were pyknotic (yellow arrows, C and D). divided (red arrows, D), shrunken (grey arrow, D) and pale (blue arrows). In subgroup C1 and subgroup C2, most of nuclei were in normal shape and size (yellow arrows, $\mathbf{E}$ and $\mathbf{F}$ ), but some neurons were darkly-stained (white arrow, F). A, B, C: scale bar $=50 \mu \mathrm{m} ; \mathrm{D}, \mathrm{E}, \mathrm{F}$ : scale bar $=20 \mu \mathrm{m}$.

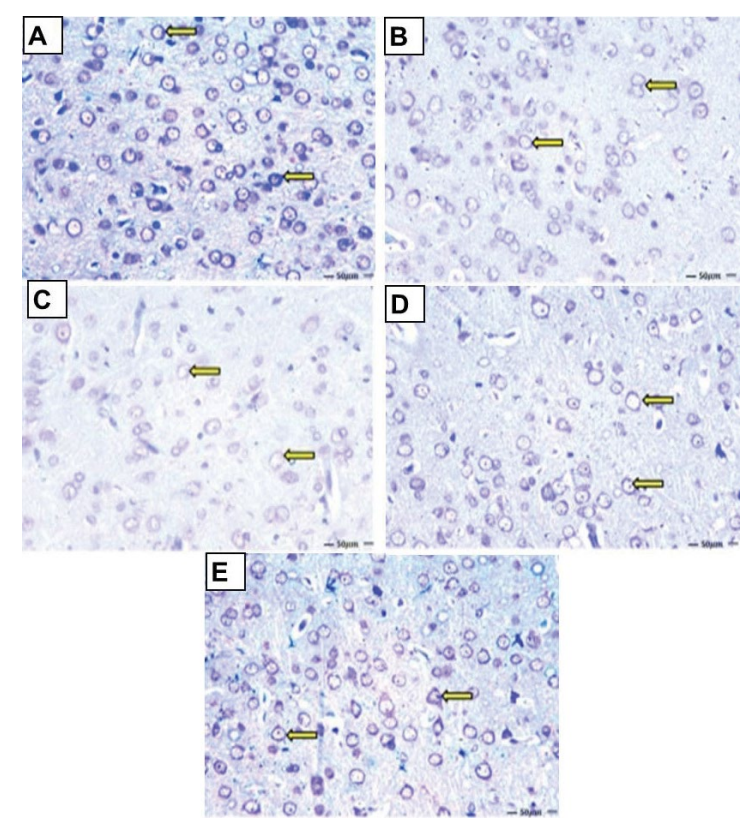

Figure 2. A-E. Representative images of toluidine blue-stained sections from rats of different groups. Nutmeg-treated group showed decrease in the density of Nissl's granules. This decrease was more obvious in subgroup B2 (yellow arrows, C) than that in subgroup B1 (yellow arrows, B). In protected group, there was increase in blue staining in the cytoplasm of the neurons. Scale bar $=50 \mu \mathrm{m}$. 




Figure 3. A-L. Representative glial fibrillary acid protein (GFAP) and caspase-3 immunostaining images of rat cerebral cortical tissues in different groups. GFAP and caspase-3 immunostaining (yellow arrows) was dramatically increased in nutmeg-treated group compared with control $(p<0.001)$. It was more obvious in subgroup B2 than that in subgroup B1. These increases were significantly reduced in protected groups. $\mathrm{N}=5$ for each group. Scale bar $=50 \mu \mathrm{m}$.


Figure 4. A-F. Representative electron microscopy images of sections from rats of different groups. Dilated rough endoplasmic reticulum (red arrows) and degenerated mitochondria (yellow arrows) were detected in nutmeg treated group (B, C), more obvious in subgroup B2. They were almost normal in protected group (E, F). Scale bar $=3000 \mu \mathrm{m}$. N $=5$ for each group.
GFAP expression in subgroups $\mathrm{C} 1$ and $\mathrm{C} 2$ compared to nutmeg-treated subgroups $(\mathrm{p}<0.001$ ) (Fig. 3A-F). In caspase-3 immunoreaction, quantitative analysis of apoptotic positive cells within the cerebral cortex revealed a significant increase in caspase-3 expression in nutmeg-treated subgroups compared with control group A $(p<0.01)$. The caspase-3 expression was significantly down-regulated after vitamin $C$ treatment (Fig. 3G-L).

\section{Electron microscopic studies}

The nerve cells of control group were rich in different organelles, such as regular nuclei, rough endoplasmic reticulum and mitochondria (Fig. 4A), while nutmeg-treated group revealed ultrastructural changes such as dilated rough endoplasmic reticulum and degenerated mitochondria with partial loss of their cristae (Fig. 4B, C). Compared to nutmeg-treated group, the neurons of the protected group showed maintenance of most of their normal structure. Most of neurons appeared normal without apparent alterations in cytoplasmic organelles. Rough endoplasmic reticulum and mitochondria were normal (Fig. 4D, E). 


\section{Agarose gel electrophoresis finding}

No apoptotic bands could be detected in the control group (lane 1). While apoptotic bands were noticed in nutmeg-treated group. They were more evident in subgroup B2 (lanes 4, 5), than that in subgroup B1 (lanes 2, 3). In the protected group, no clear apoptotic bands could be detected in subgroup C1 (lanes 6, 7), but there was decrease in apoptotic bands compared with subgroup B2 (lanes 8, 9) (Fig. 4F).

\section{DISCUSSION}

Nutmeg is considered a pro-oxidant [4]. Pro-oxidant is a chemical that can induce oxidative stress either through production of reactive oxygen species, such as free radicals, or inhibiting the anti-oxidant systems [13]. The brain is, in particular, susceptible to free radical damage due to its high level of oxygen metabolism. The brain is also relatively deficient in both free radical scavenging enzymes and endogenous antioxidants as compared to other organs [7]. This directs the attention to the antioxidants, such as vitamin $\mathrm{C}$, as protective measures for the neurotoxicity induced by nutmeg. In the present study, apoptosis was evidenced histologically by shrinkage of cell bodies and pyknotic darkly-stained nuclei. In addition, apoptotic changes were confirmed by the significant up-regulation of caspase-3 expression in neurons of nutmeg-treated group, which were more pronounced in high dose of nutmeg. Cell death in response to neurotoxins occurs as a controlled event involving a genetic programming, in which, caspase enzymes are activated [4]. Quantitative analysis of GFAP immunoreactive neurons revealed a significant increase in the number of GFAP immunoreactive neurons after low and high dose nutmeg administration compared to control. Previous studies showed that damaged neurons induce astrogliosis [17]. Moreover, in the present study, mitochondria exhibited degeneration with destroyed cristae [8]. Our gel electrophoresis results showed a marked ladder DNA fragmentation in nutmeg-treated group. This further confirms the occurrence of apoptosis. The role of antioxidants has been gaining popularity. Vitamin $\mathrm{C}$ | is considered the major water-soluble antioxidant within the body [6]. This low-molecular-weight antioxidant can safely interact with free radicals and terminate the chain reaction before vital molecules are damaged [10]. Our results showed a significant decrease in the immunoreactivity for caspase-3 of nutmeg + vitamin C treated group. The current study of animals of protected group showed, by genetic tests, great improvement in DNA injury as compared to nutmeg-treated rats. Vitamin C extract might decrease reactive oxidative species by powerful antioxidant role, thus decreasing DNA damage. Therefore, vitamin C could be a promising candidate to antagonise the harmful effects of nutmeg on the primary visual occipital cortex.

\section{CONCLUSIONS}

The results obtained from the present study revealed the toxic effects of nutmeg on the primary visual cortex in adult male albino rats. Such toxic effects were more aggravated at high-dose treatment and indicated by alterations in histological parameters. However, co-treatment with vitamin C provided a protective role against nutmeg-induced neurotoxicity through its antioxidant property.

\section{REFERENCES}

1. Adjen JO. Histological effects of chronic consumption of Nutmeg on the superior colliculus of adult Wistar rats. Int J Biomed Health Sciences. 2010; 1: 6.

2. Boada J, Cutillas B, Roig T, et al. MPP(+)-induced mitochondrial dysfunction is potentiated by dopamine. Biochem Biophys Res Commun. 2000; 268(3): 916-920, doi: 10.1006/ bbrc.2000.2232, indexed in Pubmed: 10679305.

3. De ML, Frey RJ, Longe JL. Nutmeg In project ed. Gale Encyclopedia of Alternative Medicine. Detroit, MI: Thomson Gale. 2nd Ed. 2005; 3: 1461-1463.

4. Dumri K, Letsiri S. Pro-oxidative activity in some thai spices. Acta Horticulturae. 2005(680): 25-29, doi: 10.17660/ actahortic.2005.680.2.

5. Freireich EJ, Gehan EA, Rall DP, et al. Quantitative comparison of toxicity of anticancer agents in mouse, rat, hamster, dog, monkey, and man. Cancer Chemother Rep. 1966; 50(4): 219-244, indexed in Pubmed: 4957125.

6. Halliwell B. How to characterize an antioxidant: an update. Biochem Soc Symp. 1995; 61: 73-101, indexed in Pubmed: 8660405.

7. Jyoti A, Sethi P, Sharma D. Bacopa monniera prevents from aluminium neurotoxicity in the cerebral cortex of rat brain. J Ethnopharmacol. 2007; 111(1): 56-62, doi: 10.1016/j. jep.2006.10.037, indexed in Pubmed: 17189676.

8. Kumar V, Abbas AK, Fausto N, et al. Pathological basis of disease.7th Ed. Cellular adaptation, cell injury and cell death. Elsevier Saunders. 2008; 1: 3.

9. Leuba G, Kraftsik R. Changes in volume, surface estimate, three-dimensional shape and total number of neurons of the human primary visual cortex from midgestation until old age. Anat Embryol (Berl). 1994; 190(4): 4351-4366, indexed in Pubmed: 7840422.

10. Levine M, Rumsey SC, Daruwala R, et al. Criteria and recommendations for vitamin C intake. JAMA. 1999; 281(15): 1415-1423, indexed in Pubmed: 10217058. 
11. Pavlovic V, Pavlovic D, Kocic G, et al. Ascorbic acid modulates monosodium glutamate induced cytotoxicity in rat thymus. 2009; 110(4): 205-209.

12. Peng $Y$, Kwok KHH, Yang $P H$, et al. Ascorbic acid inhibits ROS production, NF-kappa B activation and prevents ethanol-induced growth retardation and microencephaly. Neuropharmacology. 2005; 48(3): 426-434, doi: 10.1016/j. neuropharm.2004.10.018, indexed in Pubmed: 15721175.

13. Puglia CD, Powell SR. Inhibition of cellular antioxidants: a possible mechanism of toxic cell injury. Environ Health Perspect. 1984; 57: 307-311, doi: 10.1289/ehp.8457307, indexed in Pubmed: 6094175.
14. Rahman NAA, Fazilah A, Effarizah ME. Toxicity of Nutmeg (Myristicin) Food Technology Division, School of Industrial Technology, Universiti Sains Malaysia (USM). 2015: 5.

15. Robert C, Repetto, Sanjay S, et al. Snippet view. https:// books.google.com.gh/books (1996).

16. Rudgley R. The Encyclopedia of Psychoactive Substances. Thomas Dunne Books/St. Martin's Griffin Rationale of hypophsis. 1998.

17. Zhang D, Hu X, Qian Li, et al. Astrogliosis in CNS pathologies: is there a role for microglia? Mol Neurobiol. 2010; 41(2-3): 232-241, doi: 10.1007/s12035-010-8098-4, indexed in Pubmed: 20148316. 